\title{
Rehabilitation, weaning and physical therapy strategies in chronic critically ill patients
}

\author{
N. Ambrosino*,\#, E. Venturelli", G. Vagheggini* and E. Clini"
}

ABSTRACT: In critically ill patients, a prolonged hospital stay, due to the initial acute insult and adverse side-effects of drug therapy, may cause severe late complications, such as muscle weakness, prolonged symptoms, mood alterations and poor health-related quality of life.

The clinical aims of physical rehabilitation in both medical and surgical intensive care units (ICUs) are focussed on the patient to improve their short- and even long-term care.

The purpose of this article is to review the currently available evidence on comprehensive rehabilitation programmes in critically ill patients, and describe the key components and techniques used, particularly in specialised ICUs.

Despite the literature suggesting that several techniques have led to beneficial effects and that muscle training is associated with weaning success, scientific evidence is limited. Due to limitations in undertaking comparative studies in ICUs, further studies with solid clinical shortand long-term outcome measures are now welcomed.

KEYWORDS: Mechanical ventilation, physiotherapy, rehabilitation, respiratory failure, weaning

$\mathbf{P}$ rogress in treatment has markedly improved the survival of critically ill patients admitted to intensive care units (ICUs) or respiratory intermediate intensive care units (RIICUs). Increasing evidence, however, points to persisting physical disability in many survivors, associated with reduced quality of life, even years after the acute event [1-3]. Several problems may contribute to these physical limitations, including decline in pulmonary function, contractures and pressure palsies, as well as persistent muscle weakness [4], also leading to an increase in partial or complete dependence on mechanical ventilation.

\section{CONSEQUENCES OF PROLONGED MECHANICAL VENTILATION}

A long hospital stay and lack of response to or an inadequate level of appropriate therapy can lead to severe complications, such as muscle wasting and weakness, deconditioning, recurrent symptoms and mood alterations [5, 6]. "Chronic critical illness" occurs in almost half of all ICU patients with sepsis, multiple organ failure or requiring prolonged mechanical ventilation and involves muscle weakness, neuropathy, loss of lean body mass, increased adiposity and anasarca [5-7]. This condition is also associated with low serum hormone levels, catabolism [6, 8], increased prevalence of difficult-toeradicate infections [9], prolonged coma or delirium [10], skin wounds, oedema, incontinence and prolonged bed rest [11, 12].

There is a growing need for recognition of the role of rehabilitation programmes in the short- and long-term care of patients admitted to ICUs or RIICUs [13-17]. The aims of these programmes in critically ill patients are: to apply advanced costeffective therapeutic tools to decrease bed rest complications and patients' ventilator dependency; to improve residual function; to prevent the need for new hospitalisations; and to improve health status and quality of life [18-20]. Physical therapy in patients undergoing cardiac, upper abdominal and thoracic surgery may prevent and treat respiratory complications, such as secretion retention, atelectasis and pneumonia, by means of

\section{AFFILIATIONS}

*Pulmonary Rehabilitation and Weaning Center, Auxilium Vitae, Volterra,

"Pulmonary Unit, University Hospital, Pisa, and "University of Modena Reggio Emilia Ospedale Villa Pineta, Pavullo, Italy.

\section{CORRESPONDENCE}

E. Clini

University of Modena Reggio Emilia Ospedale Villa Pineta

Via Gaiato 127

41060 Pavullo

Italy

E-mail: enrico.clini@unimore.it

Received:

June 032011

Accepted after revision: Nov 042011

First published online:

Dec 012011 
different techniques. Long-term outcomes include improvement in respiratory function, reduction in re-admission to hospital and overall improvement in health status [21, 22]. Early mobilisation and maintenance of muscle strength may reduce the risk of difficult weaning, limited mobility and ventilator dependency $[23,24]$. Table 1 summarises the interventions of a rehabilitation course for critically ill patients.

\section{TREATMENT OF MUSCLE WEAKNESS}

Prolonged immobility is a contributing factor of muscle weakness in ICU patients. Therefore, passive and active mobilisation may substantially contribute to the patient's recovery in the critical care area. Mobilisation is generally delivered progressively, increasing both intensity and duration of exercise.

\section{Mobilisation}

Early mobilisation is a feasible and safe intervention that is delivered after cardio-respiratory and neurological stabilisation [25-27]. This approach, together with specific muscle training, can improve functional outcomes and cognitive and respiratory conditions [26], and reduce venous stasis and risk of deep-vein thrombosis [28]. In particular, continuous rotational therapy refers to the use of specialised beds to continuously turn patients along the longitudinal axis up to $60^{\circ}$ on each side, with pre-set degree and speed of rotation. Rather than prevention, this treatment can reduce the risk of sequential airway closure and pulmonary atelectasis, reduce the incidence rate of lower respiratory tract infection and pneumonia, and reduce the duration of endotracheal intubation and length of hospital stay [23, 30-32, 89, 90].

There is agreement on the use of early mobilisation in unconscious or sedated patients [15, 29]. This includes the use of semi-recumbent positioning with the aim being to have: the head of the bed positioned at $\geqslant 45^{\circ}[14,33]$; regular changes in postures beyond the standard 2-h turning regimen [34]; daily passive movement of all joints [91]; and (passive) bed cycling [41] and electrical stimulation as indicated [46].

\begin{tabular}{lc} 
TABLE 1 & $\begin{array}{l}\text { Rehabilitation-based activities and techniques in } \\
\text { the intensive care unit }\end{array}$ \\
Activities and techniques & \\
\hline & {$[$ Ref.] } \\
Muscle weakness & {$[15,23-29]$} \\
Passive and active-assisted mobilisation & {$[30-32]$} \\
Continuous rotational therapy & {$[18,33-40]$} \\
Postures & {$[41-43]$} \\
Active limb exercise & {$[44,45]$} \\
Peripheral muscle training & {$[46-55]$} \\
Neuromuscular electrical stimulation & {$[56-60]$} \\
Respiratory muscle training & {$[61-66]$} \\
Airway secretions & {$[67,68]$} \\
Manual hyperinflation & {$[69-75]$} \\
Percussion and vibrations & {$[76-81]$} \\
In-exsufflation & \\
Percussive ventilation & {$[82-88]$} \\
Weaning &
\end{tabular}

\section{Postures}

Prone position has been shown to result in a short-term gain in oxygenation, and improvement of ventilation and perfusion mismatch and residual lung capacity [35-38]. Improvements in lung function and reduction of lung atelectasis rate have also been seen in patients with unilateral disease when positioned with the affected lung uppermost [39, 40]. Despite their physiological rationale [18], these easy-to-apply techniques are still not widely used and it is still unclear whether the reported improvements may be associated with similar changes in stronger clinical outcomes, such as mortality.

\section{Limb exercise and peripheral muscle training}

The aims of passive, active-assisted or active-resisted limb movements are to: maintain joint range of motion; improve softtissue length and muscle strength; and decrease the risk of thromboembolism [92]. Quadriceps strength and functional status showed no difference in patients where early mobilisation was added to standard chest physiotherapy compared with physiotherapy alone. However, the total walked distance, the isometric quadriceps force and the perceived functional wellbeing were significantly better in those patients with early mobilisation [41]. A gradual mobility protocol for both upper and lower limbs was then introduced. It was found to be feasible and safe and led to a decreased length of hospital stay in patients requiring mechanical ventilation [42]. In particular, addition of a supported arm-training protocol was effective in patients recently weaned from mechanical ventilation and admitted to an RIICU [43].

Muscle mass and the ability to perform aerobic exercise declines with inactivity [93]. In critically ill and complex patients, skeletal muscle training aims at improving strength, thus, increasing the patient's ability to recover activities of daily life [26], improving hospital stay and survival. In patients on long-term mechanical ventilation and those who are difficult-to-wean, a tailored training programme seems to be effective in speeding up weaning time and improving hospital stay and survival [44].

Prolonged inactivity is more likely to cause skeletal muscle dysfunction and atrophy in anti-gravity muscles, with reduced capacity to perform aerobic exercise [94]. In severely disabled patients, peripheral muscle training (lifting weights or pushing against a resistance with the limbs) produces a specific gain in strength and recovery of activities of daily life, although evidence of effects after an episode of acute respiratory failure has not yet been defined [45]. Figure 1 illustrates examples of active and passive peripheral muscle activities in bed-bound critically ill patients.

\section{Neuromuscular electrical stimulation}

Neuromuscular electrical stimulation (NMES) can induce changes in muscle function without any form of ventilatory stress [47]. NMES can be easily performed in the ICU and applied to the lower limb muscles of patients lying in bed. Nevertheless, to date, no clinical studies have completely demonstrated the additional effect of NMES on exercise tolerance when compared with conventional training. Patients with chronic obstructive pulmonary disease (COPD) [48, 49] or congestive heart failure [50] are more likely to benefit; moreover, NMES has also been considered as a means to prevent the ICU neuromyopathy [51]. Although NMES may have a role, the timing of such an 


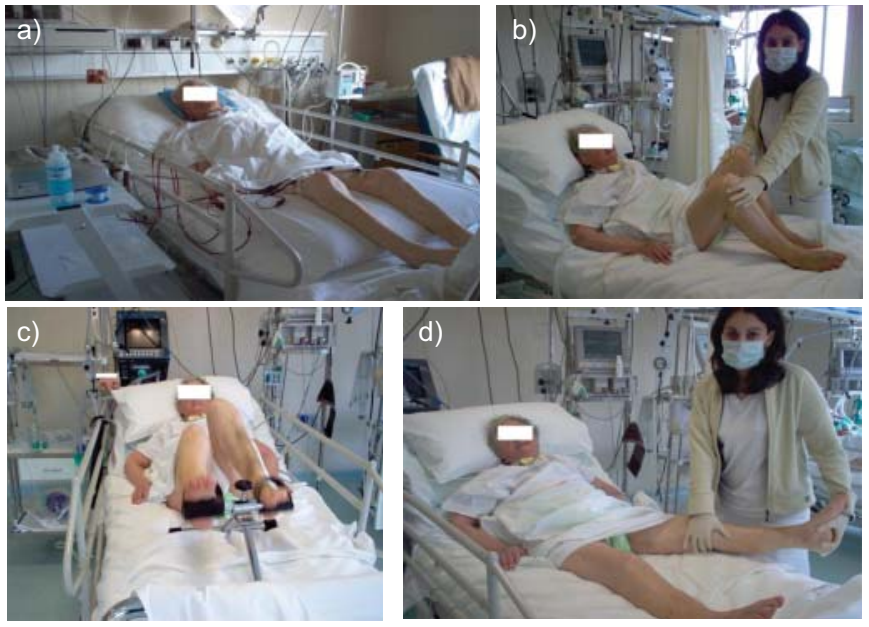

FIGURE 1. Examples of a) passive, b, d) active-assisted and c) active peripheral muscle activities in bed-bound critically ill patients.

intervention has yet to be determined. An anabolic stimulus early on in critical illness may have little benefit, as shown by the lack of NMES effect in patients with septic shock [52]. Nevertheless the severity of the acute illness also appears to have an important role, as the benefit of NMES has been shown in patients with sepsis without shock [53]. This is supported by the basic scientific literature. An anabolic stimulus at a time when mitochondrial dysfunction is present [54] may not be beneficial, although survival appears to be associated with early activation of mitochondrial biogenesis [55], which could be stimulated by NMES.

\section{Respiratory muscle training}

Weakness of the respiratory muscle mass, imbalance between respiratory muscle strength and load of the respiratory system, and cardiovascular impairment are major determinants of weaning failure in ventilated individuals. In ICU patients, these factors and the excessive use (especially in the past) of controlled mechanical ventilation may rapidly lead to diaphragmatic dysfunction [95]. Nevertheless, the rationale of respiratory muscle training in critically ill patients is still controversial and is not part of mainstream practice in many institutions. Indeed, the diaphragm of patients with COPD is as effective as that of normal subjects in generating pressure at comparable lung volumes [96]. An adaptive change toward the slow-to-fast characteristics (resistance to fatigue) of the muscle fibres due to increased operational lung volume has been shown [97]. Recent literature debates the potential role of inspiratory muscle training as a component of pulmonary rehabilitation in severely disabled COPD and neuromuscular disease patients [56, 57], aimed at improving their strength and reducing the respiratory system load perception. Notwithstanding, studies in ventilator dependent COPD patients have shown that respiratory muscle training may be associated with a favourable weaning outcome [58-60].

\section{MANAGEMENT OF AIRWAY SECRETIONS}

Increased retention of bronchial secretion, as a result of mucociliary dysfunction, and reduced cough function, as a result of inspiratory and expiratory muscle weakness, cause an increased risk of nosocomial pneumonia [9]. Chest physiotherapy should prevent such complications by improving ventilation and gas exchange, and by reducing airway resistance and work of breathing [16]. Several manually assisted techniques (manual hyperinflation and percussions/vibrations) and mechanical devices are often applied to facilitate the movement of excess mucus (table 1).

\section{Manual hyperinflation}

This technique is aimed at preventing pulmonary collapse (or re-expanding collapsed alveoli), improving oxygenation and lung compliance, and facilitating the movement of secretions toward the central airways [61]. The guidelines for the application of manual hyperinflation vary across units. The possible physiological side-effects of delivered air volume, flow rates and airway pressure must be carefully considered, especially in patients on mechanical ventilation [62-64]. An increase in air volume with this technique can be obtained both manually or with assisted mechanical ventilation, with each producing similar benefits in clearing excessive mucus $[65,66]$.

\section{Percussion and vibrations}

Manual clapping over a selected area of the chest wall and vibration that involves compression of the chest during the expiratory phase of ventilation are common techniques used to increase airway clearance by shifting secretions from the periphery airways towards the central airways. The techniques may be applied along with the use of gravity by positioning the patients to assist with drainage of secretions. Currently, in critical ventilated patients with normal cough competence, increase of mucus clearance is described without a significant change of blood gases and lung compliance [16, 67, 68].

\section{In-exsufflation}

This is the most popular mechanically assisted technique adopted to promote removal of excessive mucus in neuromuscular disease [69]. In-exsufflation is also referred to as cough assist and is usually limited to patients with neuromuscular and skeletal conditions who have a weak cough (flow rate $<250 \mathrm{~L} \cdot \mathrm{min}^{-1}$ ). It acts by inflating the airways with a large volume of air that is rapidly exsufflated by negative pressure, thus simulating the physiological mechanism of cough [70-72]. The safety and clinical advantage (avoidance or delay of tracheostomy and/or endotracheal intubation) of this device when compared with conventional chest physiotherapy has been shown in hospitalised neuromuscular patients with recent upper respiratory tract infection $[73,74]$. The usefulness of this technique in allowing for extubation in patients judged as needing tracheostomy has been recently reported [75].

\section{Intrapulmonary percussive ventilation}

This mechanical device creates a percussive effect in the airways, thus facilitating mucus clearance through direct highfrequency oscillatory ventilation that is able to help the alveolar recruitment [76]. Positive effects from this technique have been shown during both acute and chronic phases in patients with respiratory distress [77], neuromuscular diseases [78] and pulmonary atelectasis [79]. In hospitalised COPD patients with respiratory acidosis, this technique has also been shown to prevent the deterioration of the acute episode, thus avoiding endotracheal intubation [80]. In tracheostomised patients recently weaned from mechanical ventilation, the addition of intermittent percussive ventilation to usual chest 
physiotherapy was associated with improvement of oxygenation and expiratory muscle performance also leading to a substantial reduction in the risk of late onset pneumonia [81].

\section{WEANING}

COPD patients can have substantial difficulties in completing the weaning process due to the pulmonary/thoracic (imbalance between capacity of and load on respiratory system) and systemic involvement of their chronic disease [98]. Among the systemic factors, immobility (even by neuromuscular blocking agents), muscle de-conditioning (with muscle disuse atrophy), systemic use of corticosteroids, malnutrition and gas exchange abnormalities are common obstacles to weaning in critically ill patients [6, 99]. For these patients, difficult/prolonged weaning requires a rehabilitation-based, specific process aimed at restoring the individual's independence from the ventilator.

\section{Therapist-driven protocols}

Despite studies being inconclusive in determining whether decreasing pressure support or T-piece tube trials are the best method to speed up the weaning process $[82,100]$, recent trials have underlined the role of a standardised protocol (the so-called therapist-driven protocol; TDP) to resume the patient's spontaneous breathing more easily. TDPs represent ICU staff consensus summarised into a daily care plan (algorithm), essentially based on reporting day by day changes in patient to ventilator interactions (e.g. the change of the ventilatory setting) [83]. The role of professional expertise in guiding this process is crucial.

At present there are no definitive results regarding the application of TDP as a fixed protocol-based procedure to discontinue mechanical ventilation [84], the use of this care plan has been proven to be effective when applied to the weaning process in the critical care area. SAURA et al. [85] prospectively studied 51 patients weaned using the TDP and found that they required less mechanical ventilation and spent less time in hospital compared with retrospective controls. These results were confirmed by a randomised controlled study by ELY et al. [86] who demonstrated that the use of TDP for weaning reduces costs and clinical complications compared to conventional ventilation. Overall, the clinical experience in this field seems to confirm positive results regarding the weaning process. In fact, when using TDP, a weaning success rate of $\sim 60 \%$ was reported in complex and difficult-to-wean patients [87, 88]. Finally, increasing periods of spontaneous breathing (T-piece) trials or decreasing levels of pressure support were found to be equally effective during TDP in tracheostomised and difficultto-wean COPD patients [101]. Despite the evidence, recent surveys have shown that TDPs are unevenly or infrequently adopted in ICUs in different countries [100-103].

\section{CONCLUSION}

Due to an increasing number of ICU admissions worldwide, which all carry a risk of subsequent complications and mortality over the following years $[3,104]$, comprehensive programmes including physiotherapy should be implemented to speed up the patients' functional recovery and to prevent the complications of prolonged immobilisation, especially in ventilatordependent or difficult-to-wean patients [19, 104]. To manage the multiple and complex problems of these patients, integrated programmes dealing with both whole-body physical therapy and pulmonary care are needed $[14,15]$.
To date, the application of both techniques and strategies is more likely to be dedicated to those critically ill patients who are specifically admitted to an RIICU. Indeed, there is still limited scientific evidence to support such a comprehensive approach to all critically ill patients. Therefore, despite the ethical difficulties experienced with randomised control trials and other reasons which make studies undertaken in ICUs challenging, we need randomised studies with solid clinical short- and longterm outcome measures.

\section{STATEMENT OF INTEREST}

None declared.

\section{REFERENCES}

1 Epstein SK. Size of the problem, what constitutes prolonged mechanical ventilation, natural history, epidemiology. In: Ambrosino N, Goldstein RS, eds. Ventilatory Support in Chronic Respiratory Failure. New York, Informa, 2008; pp. 39-57.

2 Kahn JM, Benson NM, Appleby D, et al. Long-term acute care hospital utilization after critical illness. JAMA 2010; 303: 2253-2259.

3 Herridge MS, Tansey CM, Matté A, et al. Functional disability 5 years after acute respiratory distress syndrome. $N$ Engl J Med 2011; 364: 1293-1304.

4 Zanni JM, Korupolu R, Fan E, et al. Rehabilitation therapy and outcomes in acute respiratory failure: an observational pilot project. J Crit Care 2010; 25: 254-262.

5 Nelson JE, Cox CE, Hope AA, et al. Concise clinical review: chronic critical illness. Am J Respir Crit Care Med 2010; 182: 446-454.

6 Ambrosino N, Gabbrielli L. The difficult-to-wean patient. Expert Rev Respir Med 2010; 4: 685-692.

7 Hollander JM, Mechanick JI. Nutrition support and the chronic critical illness syndrome. Nutr Clin Pract 2006; 21: 587-604.

8 Van den Berghe G, de Zegher F, Veldhuis JD, et al. Thyrotrophin and prolactin release in prolonged critical illness: dynamics of spontaneous secretion and effects of growth hormones ecretagogues. Clin Endocrinol 1997; 47: 599-612.

9 Kalb TH, Lorin S. Infection in the chronically critically ill: unique risk profile in a newly defined population. Crit Care Clin 2002; 18 : 529-552.

10 Nelson JE, Tandon N, Mercado AF, et al. Brain dysfunction: another burden for the chronically critically ill. Arch Intern Med 2006; 166: 1993-1999.

11 Carasa M, Polycarpe M. Caring for the chronically critically ill patient: establishing a wound-healing program in a respiratory care unit. Am J Surg 2004; 188: 18-21.

12 De Jonghe B, Bastuji-Garin S, Durand MC, et al. Respiratory weakness is associated with limb weakness and delayed weaning in critical illness. Crit Care Med 2007; 35: 2007-2015.

13 Norrenberg M, Vincent JL. A profile of European intensive care unit physiotherapists. Intensive Care Med 2000; 26: 988-994.

14 Gosselink R, Bott J, Johnson M, et al. Physiotherapy for adult patients with critical illness: recommendations of the European Respiratory Society and European Society of Intensive Care Medicine Task Force on Physiotherapy for Critically Ill Patients. Intensive Care Med 2008; 34: 1188-1199.

15 Hanekom S, Gosselink R, Dean E, et al. The development of a clinical management algorithm for early physical activity and mobilization of critically ill patients: synthesis of evidence and expert opinion and its translation into practice. Clin Rehabil 2011; 25: 771-787.

16 Stiller K. Physiotherapy in intensive care. Towards an evidencebased practice. Chest 2000; 118: 1801-1813.

17 Scala R, Corrado A, Confalonieri M, et al. Increased number and expertise of Italian respiratory high-dependency care units: the second national survey. Respir Care 2011; 56: 1100-1107. 
18 Novoa N, Ballesteros E, Jiménez MF, et al. Chest physiotherapy revisited: evaluation of its influence on the pulmonary morbidity after pulmonary resection. Eur J Cardiothorac Surg 2011; 40: 130-134.

19 Carpenè N, Vagheggini G, Panait E, et al. A proposal of a new model for long-term weaning: respiratory intensive care unit and weaning center. Respir Med 2010; 104: 1505-1511.

20 Montagnani G, Vagheggini G, Panait Vlad E, et al. Use of the functional independence measure following a weaning program from mechanical ventilation in difficult to wean patients. Phys Ther 2011; 9: 1109-1115.

21 Ambrosino N, Gabbrielli L. Physiotherapy in the perioperative period. Best Pract Res Clin Anaesthesiol 2010; 24: 283-289.

22 Ambrosino N, Janah N, Vagheggini G. Fisioterapia en pacientes gravemente doentes [Physiotherapy in critically ill patients]. Rev Port Pneumol 2011; 17: 283-288.

23 Clini E, Ambrosino N. Early physiotherapy in the respiratory intensive care unit. Respir Med 2005; 99: 1096-1104.

24 Pohlman MC, Schweickert WD, Pohlman AS, et al. Feasibility of physical and occupational therapy beginning from initiation of mechanical ventilation. Crit Care Med 2010; 38: 2089-2094.

25 Thomsen GE, Snow GL, Rodriguez L, et al. Patients with respiratory failure increase ambulation after transfer to an intensive care unit where early activity is a priority. Crit Care Med 2008; 36: 1119-1124.

26 Schweickert WD, Pohlman MC, Pohlman AS, et al. Early physical and occupational therapy in mechanically ventilated, critically ill patients: a randomised controlled trial. Lancet 2009; 373: 1874-1882.

27 Thomas AJ. Physiotherapy led early rehabilitation of the patient with critical illness. Phys Ther Rev 2011; 16: 46-57.

28 Partsch H. Bed rest versus ambulation in the initial treatment of patients with proximal deep vein thrombosis. Curr Opin Pulm Med 2002; 8: 389-393.

29 Kress JP. Clinical trials of early mobilization of critically ill patients. Crit Care Med 2009; 37: Suppl. 10, S442-S447.

30 Gentilello L, Thompson DA, Tonnesen AS, et al. Effect of a rotating bed on the incidence of pulmonary complications in critically ill patients. Crit Care Med 1988; 16: 783-786.

31 Fink MP, Helsmoortel CM, Stein KL, et al. The efficacy of an oscillating bed in the prevention of lower respiratory tract infection in critically ill victims of blunt trauma. Chest 1990; 97: 132-137.

32 Kirschenbaum L, Azzi E, Sfeir T, et al. Effect of continuous rotational therapy on the prevalence of ventilator-associated pneumonia in patients requiring long-term ventilatory care. Crit Care Med 2002; 30: 1983-1986.

33 Dodek P, Keenan S, Cook D, et al. Evidence-based clinical practice guideline for the prevention of ventilator-associated pneumonia. Ann Intern Med 2004; 14: 305-313.

34 Krishnagopalan S, Johnson EW, Low LL, et al. Body positioning of intensive care patients: clinical practice versus standards. Crit Care Med 2002; 30: 2588-2592.

35 Gattinoni L, Tognoni G, Pesenti A, et al. Prone-Supine Study Group. Effect of prone positioning on the survival of patients with acute respiratory failure. N Engl J Med 2001; 345: 568-573.

36 Mure M, Martling C-R, Lindahl SG. Dramatic effect on oxygenation in patients with severe acute lung insufficiency treated in the prone position. Crit Care Med 1997; 25: 1539-1544.

37 Jolliet P, Bulpa P, Chevrolet JC. Effects of the prone position on gas exchange and hemodynamics in severe acute respiratory distress syndrome. Crit Care Med 1998; 26: 1539-1544.

38 Chatte G, Sab JM, Dubois JM, et al. Prone positioning in mechanically ventilated patients with severe acute respiratory failure. Am J Respir Crit Care Med 1997; 155: 473-478.

39 Gillespie DJ, Rehder K. Body position and ventilation-perfusion relationships in unilateral pulmonary disease. Chest 1987; 91: 75-79.

40 Stiller K, Jenkins S, Grant R. Acute lobar atelectasis: a comparison of five physiotherapy regimens. Physiother Theory Pract 1996; 12: 197-209.
41 Burtin C, Clerckx B, Robbeets C, et al. Early exercise in critically ill patients enhances short-term functional recovery. Crit Care Med 2009; 37: 2499-2505.

42 Morris PE, Goad A, Thompson C, et al. Early intensive care unit mobility therapy in the treatment of acute respiratory failure. Crit Care Med 2008; 36: 2238-2243.

43 Porta R, Vitacca M, Gilè LS, et al. Supported arm training in patients recently weaned from mechanical ventilation. Chest 2005; 128: 2511-2520.

44 Clini EM, Crisafulli E, Antoni FD, et al. Functional recovery following physical training in tracheotomized and chronically ventilated patients. Respir Care 2011; 56: 306-313.

45 Nava S. Rehabilitation of patients admitted to a respiratory intensive care unit. Arch Phys Med Rehabil 1998; 79: 849-854.

46 Gerovasili V, Stefanidis K, Vitzilaios K, et al. Electrical muscle stimulation preserves the muscle mass of critically ill patients: a randomized study. Crit Care 2009; 13: R161.

47 Ambrosino N, Strambi S. New strategies to improve exercise tolerance in chronic obstructive pulmonary disease. Eur Respir J 2004; 24: 313-322.

48 Zanotti E, Felicetti G, Maini M, et al. Peripheral muscle strength training in bed-bound patients with COPD receiving mechanical ventilation. Effect of electrical stimulation. Chest 2003; 124: 2992-2996.

49 Vivodtzev I, Pépin JL, Vottero G, et al. Improvement in quadriceps strength and dyspnea in daily tasks after 1 month of electrical stimulation in severely deconditioned and malnourished COPD. Chest 2006; 129: 1540-1548.

50 Nuhr MJ, Pette D, Berger R, et al. Beneficial effects of chronic low-frequency stimulation of thigh muscle in patients with advanced chronic heart failure. Eur Heart J 2004; 25: 136-143.

51 Routsi C, Gerovasili V, Vasileiadis I, et al. Electrical muscle stimulation prevents critical illness polyneuromyopathy: a randomized parallel intervention trial. Crit Care 2010; 14: R74.

52 Poulsen JB, Møller K, Jensen CV, et al. Effect of transcutaneous electrical muscle stimulation on muscle volume in patients with septic shock. Crit Care Med 2011; 39: 456-461.

53 Rodriguez PO, Setten M, Maskin LP, et al. Muscle weakness in septic patients requiring mechanical ventilation: protective effect of transcutaneous neuromuscular electrical stimulation. J Crit Care 2011; [Epub ahead of print DOI: 10.1016/j.jcrc.2011.04.010]

54 Brealey D, Karyampudi S, Jacques TS, et al. Mitochondrial dysfunction in a long-term rodent model of sepsis and organ failure. Am J Physiol Regul Integr Comp Physiol 2004; 286: R491-R497.

55 Carré JE, Orban JC, Re L, et al. Survival in critical illness is associated with early activation of mitochondrial biogenesis. Am J Respir Crit Care Med 2010; 182: 745-751.

56 Gosselink R, De Vos J, van den Heuvel SP, et al. Impact of inspiratory muscle training in patients with COPD: what is the evidence? Eur Respir J 2011; 37: 416-425.

57 American Thoracic Society. Respiratory care of the patient with Duchenne muscular dystrophy: ATS consensus statement. Am J Respir Crit Care Med 2004; 170: 456-465.

58 Martin AD, Davenport PD, Franceschi AC, et al. Use of inspiratory muscle strength training to facilitate ventilator weaning. Chest 2002; 122: 192-196.

59 Aldrich TK, Karpel JP, Uhrlass RM, et al. Weaning from mechanical ventilation: adjunctive use of inspiratory muscle training. Crit Care Med 1989; 17: 14-19.

60 Moodie LH, Reeve JC, Vermeulen N, et al. Inspiratory muscle training to facilitate weaning from mechanical ventilation: protocol for a systematic review. BMC Res Notes 2011; 4: 283.

61 Denehy L. The use of manual hyperinflation in airway clearance. Eur Respir J 1999; 14: 958-965.

62 Clarke RC, Kelly BE, Convery PN, et al. Ventilatory characteristics in mechanically ventilated patients during manual hyperventilation for chest physiotherapy. Anesthesia 1999; 54: 936-940. 
63 Singer M, Vermaat J, Hall G, et al. Hemodynamic effects of manual hyperinflation in critically ill mechanically ventilated patients. Chest 1994; 106: 1182-1187.

64 Turki M, Young MP, Wagers SS, et al. Peak pressures during manual ventilation. Respir Care 2005; 50: 340-344.

65 Maa SH, Hung TJ, Hsu KH, et al. Manual hyperinflation improves alveolar recruitment in difficult-to-wean patients. Chest 2005; 128: 2714-2721.

66 Stiller K, Geake T, Taylor J, et al. Acute lobar atelectasis: a comparison of two chest physiotherapy regimens. Chest 1990; 98: 1336-1340.

67 Ntoumenopoulos G, Presneill JJ, McElholum M, et al. Chest physiotherapy for the prevention of ventilator-associated pneumonia. Intensive Care Med 2002; 28: 850-856.

68 Eales CJ, Barker M, Cubberley NJ. Evaluation of a single chest physiotherapy treatment to post-operative, mechanically ventilated cardiac surgery patients. Physiother Theory Pract 1995; 11: 23-28.

69 Ambrosino N, Carpenè N, Gherardi M. Chronic respiratory care in neuromuscular diseases for adults. Eur Respir J 2009; 34: 444-451.

70 Bach JR. Mechanical insufflation-exsufflation: a comparison of peak expiratory flows with manually assisted and unassisted coughing techniques. Chest 1993; 104: 1553-1562.

71 Bach JR. Update and perspective on noninvasive respiratory muscle aids. Part 2. The expiratory aids. Chest 1994; 105: 1538-1544.

72 Winck JC, Gonçalves MR, Lourenço C, et al. Effects of mechanical insufflation-exsufflation on respiratory parameters for patients with chronic airway secretion encumbrance. Chest 2004; 126: 774-780.

73 Vianello A, Corrado A, Arcaro G, et al. Mechanical insufflationexsufflation improves outcomes for neuromuscular disease patients with respiratory tract infections. Am J Phys Med Rehabil 2005; 84: 83-88.

74 Branson RD. Secretion management in the mechanically ventilated patient. Respir Care 2007; 52: 1328-1347.

75 Bach JR, Gonçalves MR, Hamdani I, et al. Extubation of patients with neuromuscular weakness: a new management paradigm. Chest 2010; 137: 1033-1039.

76 Salim A, Martin M. High frequency percussive ventilation. Crit Care Med 2005; 33: Suppl. 3, S241-S245.

77 Velmahos GC, Chan LS, Tatevossian R, et al. High-frequency percussive ventilation improves oxygenation in patients with ARDS. Chest 1999; 116: 440-446.

78 Crescimanno G, Marrone O. High frequency chest wall oscillation plus mechanical in-exsufflation in Duchenne muscular dystrophy with respiratory complications related to pandemic Influenza A/H1N1. Rev Port Pneumol 2010; 16: 912-916.

79 Tsuruta R, Kasaoka S, Okabayashi K, et al. Efficacy and safety of intrapulmonary percussive ventilation superimposed on conventional ventilation in obese patients with compression atelectasis. J Crit Care 2006; 2: 328-332.

80 Vargas F, Boyer A, Bui HN, et al. Effect of intrapulmonary percussive ventilation on expiratory flow limitation in chronic obstructive pulmonary disease patients. J Crit Care 2009; 24: 212-219.

81 Clini EM, Antoni FD, Vitacca M, et al. Intrapulmonary percussive ventilation in tracheostomized patients: a randomized controlled trial. Intensive Care Med 2006; 32: 1994-2001.

82 Esteban A, Frutos F, Tobin M, et al. A comparison of four methods of weaning from mechanical ventilation. $N$ Engl J Med 1995; 332: 345-350.

83 Vitacca M. Therapist driven protocols. Monaldi Arch Chest Dis 2003; 59: 342-344.

84 Krishnan JA, Moore D, Robeson C, et al. A prospective, controlled trial on a protocol-based strategy to discontinue mechanical ventilation. Am J Respir Crit Care Med 2004; 169: 673-678.
85 Saura P, Blanch L, Mestre L, et al. Clinical consequences of the implementation of a weaning protocol. Intensive Care Med 1996; 22: 1052-1056.

86 Ely EW, Baker AM, Dunagan BP, et al. Effect of the duration of mechanical ventilation of identifying patients capable of breathing spontaneously. N Engl J Med 1996; 335: 1864-1869.

87 Sheinhorn DJ, Chao DC, Stearn-Hassenpflug M, et al. Post-ICU mechanical ventilation treatment of 1123 patients at a regional weaning center. Chest 1997; 111: 1654-1659.

88 Rose L, Blackwood B, Burns SM, et al. International perspectives on the influence of structure and process of weaning from mechanical ventilation. Am J Crit Care 2011; 20: e10-e18.

89 Raoof S, Chowdhrey N, Raoof S, et al. Effect of combined kinetic therapy and percussion therapy on the resolution of atelectasis in critically ill patients. Chest 1999; 7: 1658-1666.

90 de Boisblanc BP, Castro M, Everret B, et al. Effect of airsupported, continuous, postural oscillation on the risk of early ICU pneumonia in non-traumatic critical illness. Chest 1993; 103: 1543-1547.

91 Clavet H, Hebert PC, Fergusson D, et al. Joint contracture following prolonged stay in the intensive care unit. CMAJ 2008; 178: 691-697.

92 Koch SM, Fogarty S, Signorino C, et al. Effect of passive range motion on intracranial pressure in neurosurgical patients. J Crit Care 1996; 11: 176-179.

93 Bloomfield SA. Changes in musculoskeletal structure and function with prolonged bed rest. Med Sci Sport Exerc 1997; 29: 197-206.

94 Coyle EF, Martin WH 3rd, Bloomfield SA, et al. Effects of detraining on response to sub maximal exercise. J Appl Physiol 1985; 59: 853-859.

95 Le Bourdelles G, Viires N, Boczkowski J, et al. Effects of mechanical ventilation on diaphragmatic contractile properties in rats. Am J Respir Crit Care Med 1994; 149: 1539-1544.

96 Similowski T, Yan S, Gauthier AP, et al. Contractile properties of the human diaphragm during chronic hyperinflation. $N$ Engl J Med 1991; 325: 917-923.

97 Levine S, Kaiser L, Leferovich J, et al. Cellular adaptations in the diaphragm in chronic obstructive pulmonary disease. $N$ Engl J Med 1997; 337: 1799-1806.

98 Peñuelas O, Frutos-Vivar F, Fernández C, et al. Characteristics and outcomes of ventilated patients according to time to liberation from mechanical ventilation. Am J Respir Crit Care Med 2011; 184: 430-437.

99 MacIntyre NR, Cook DJ, Ely EW, et al. Evidence based guidelines for weaning and discontinuing ventilatory support: a collective task force facilitated by the American College of Chest Physicians; the American Association for Respiratory Care; and the American College of Critical Care Medicine. Chest 2001; 120: Suppl. 6, 375s-395s.

100 Brochard L, Rauss A, Benito S, et al. Comparison of three methods of gradual withdrawal from ventilatory support during weaning from mechanical ventilation. Am J Respir Crit Care Med 1994; 150: 896-903.

101 Schonhofer B, Euteneuer S, Nava S, et al. Survival of mechanically ventilated patients admitted to a specialised weaning centre. Intensive Care Med 2002; 28: 908-916.

102 Vitacca M, Vianello A, Colombo D, et al. Comparison of two methods for weaning COPD patients requiring mechanical ventilation for more than 15 days. Am J Respir Crit Care Med 2001; 164: 225-230.

103 Stoller JK, Xu M, Mascha E, et al. Long term outcomes for patient discharged from a long term hospital-based weaning unit. Chest 2003; 124: 1892-1899.

104 Martin UJ, Hincapie L, Nimchuk M, et al. Impact of whole-body rehabilitation in patients receiving chronic mechanical ventilation. Crit Care Med 2005; 33: 2259-2265. 